\title{
A rabbit model of implant-related osteomyelitis inoculated with biofilm after open femoral fracture
}

\author{
XIANG ZHANG $^{1,2}$, YUN-FEI MA ${ }^{1,2}$, LEI WANG ${ }^{1,2}$, NAN JIANG ${ }^{1,2}$, \\ CHENG-HE QIN ${ }^{1}$, YAN-JUN HU ${ }^{1}$ and BIN YU ${ }^{1,2}$ \\ ${ }^{1}$ Department of Orthopaedics and Traumatology; ${ }^{2}$ Guangdong Provincial Key Laboratory of Bone and Cartilage \\ Regenerative Medicine, Nanfang Hospital, Southern Medical University, Guangzhou, Guangdong 510515, P.R. China
}

Received March 1, 2017; Accepted August 30, 2017

DOI: $10.3892 / \mathrm{etm} .2017 .5138$

\begin{abstract}
Currently, animal models used in research on implant-associated osteomyelitis primarily use intramedullary fixation and initial inoculum of planktonic bacterial cells. However, these techniques have certain limitations, including lack of rotational stability and instable inoculation. To improve these models, the present study aimed to establish a novel rabbit model of implant-associated osteomyelitis using biofilm as the initial inoculum following plate fixation of the femoral fracture. A total of 24 New Zealand White rabbits were randomly divided into two equal groups. Osteotomy was performed at the right femoral shaft using a wire saw following fixation with a 5-hole stainless steel plate. The plates were not colonized with bacteria in group 1, but colonized with a biofilm of Staphylococcus aureus (American Type Culture Collection, 25923 ) in group 2 . All the rabbits were sacrificed after 21 days for clinical, X-ray, micro-computed tomography and histological assessments of the severity of osteomyelitis. Scanning electron microscopy and confocal laser scanning microscopy were used for biofilm assessment. In group 2, pus formation, periosteal reaction, cortical destruction and absorption were observed in all the rabbits and biofilm formation was observed on all the plates. However, no pus formation was observed except for a slight inflammatory response and all the plates appeared clean without infection in group 1 . The differences between the two groups were statistically significant regarding histologic scores and semi-quantification of the bacteria on the plates $(\mathrm{P}<0.001)$. In the present study, a novel rabbit model of infection following internal plate fixation of open fracture was successfully established, providing a novel tool for the study of implant-associated osteomyelitis.
\end{abstract}

Correspondence to: Professor Bin Yu or Professor Yan-Jun Hu, Department of Orthopaedics and Traumatology, Nanfang Hospital, Southern Medical University, 1838 Guangzhou Avenue North, Guangzhou, Guangdong 510515, P.R. China

E-mail: yubinhot@163.com

E-mail: huyanjun1212@163.com

Key words: animal model, biofilm, Staphylococcus aureus, femur fracture, osteomyelitis

\section{Introduction}

Implant-related osteomyelitis is a significant post-operation complication for orthopedic or orthopedic trauma patients undergoing fracture fixation. Infections associated with medical devices accounted for over $70 \%$ of all the orthopedic infections (1), leading to infected non-union or osteomyelitis with limited treatment options and a heavy socio-economic impact (2).

Animal models are one of the most commonly used, effective and valuable tools for research on implant-related osteomyelitis. The model with intramedullary fixation proposed by Worlock et al has been widely employed (3), but the intramedullary (IM) nail described by Worlock lacks rotational stability, differing from clinical cases where interlocking bolts are used (4).

In addition, at least $65 \%$ of all infections are caused by the biofilm bacteria in the developed world $(5,6)$. Staphylococcal species are found to be the most prevalent etiological agents of orthopedic infections, representing $75.3 \%$ of all strains with $\mathrm{S}$. aureus generally exhibiting the highest prevalence (35.5\% overall prevalence) (7). As a result of biofilm formation on the implant surface, however, antibiotic treatment of the staphylococcal species infections is often faced with failure (8). The animal models of osteomyelitis used to use planktonic bacterial cells as initial inoculum, but with the advent of bacterial biofilm concept, the limitation of planktonic bacterial inoculum and the important role that biofilm bacteria play in osteomyelitis have been perceived (9). Thus, a new model of implant-related osteomyelitis has been proposed wherein a well-established, mature biofilm is used as initial inoculum instead of planktonic bacterial cells (10).

As a general rule, animal models should reflect the clinical situation as much as possible for basic research on new therapeutic options. Therefore, in order to improve the above-mentioned problems, we aimed to establish a rabbit model of osteomyelitis following plate fixation of femoral fracture with initial inoculation by bacterial biofilm.

\section{Materials and methods}

Animals. A total of 24 male New Zealand White rabbits (3.0-3.5 kg in weight; six months in average age) were randomly 
divided into two groups. Group $2(n=12)$ treated with biofilm served as positive controls of infection and group $1(n=12)$ treated without biofilm as negative controls of infection. All animals were kept in a single cage, supplied plenty food and water. All operations were performed under good anesthesia. All procedures performed in studies involving animals were in accordance with the ethical standards of our institution's Institutional Animal Care and Oversight Committee (Project SYXK No. 2015-0056, Southern Medical University, Guangzhou, Guangdong, China).

Bacterial and implants. Staphylococcus aureus (ATCC 25923) was provided by the Infectious Diseases Department of Nanfang Hospital; 316L stainless steel plates (35 $\mathrm{mm}$ in length, $6.5 \mathrm{~mm}$ in width and $2.0 \mathrm{~mm}$ in thickness) with 5 holes were used for fracture fixation. Four screws (10 $\mathrm{mm}$ in length and $2.4 \mathrm{~mm}$ in diameter) were used to fix each implant.

Biofilm growth. S. aureus was prepared by an overnight culture in Luria-Bertani (LB) broth. Then the bacterial concentration was adjusted to an $\mathrm{OD}_{600 \mathrm{~nm}}$ of 0.5 , corresponding to $10^{8} \mathrm{CFU} / \mathrm{ml}$ by a microplate reader (SpectraMax M5, Molecular Devices, USA). The concentration of $S$. aureus in the inoculum and the OD suspension was confirmed by colony forming unit (CFU) quantification of serially diluted suspensions on agar. The bacterial suspension was then diluted 100 -fold to $10^{6} \mathrm{CFU} / \mathrm{ml}$. Next, $5 \mathrm{ml}$ of bacterial solution and a piece of steel plate were placed into a $15 \mathrm{ml}$ centrifuge tube, and then the tube was incubated for $48 \mathrm{~h}$ at $37^{\circ} \mathrm{C}$ with shaking $(200 \mathrm{rpm})$ by a constant temperature shaking incubator. Finally, the plate was taken out from the tube and washed 3 times with phosphate buffer solution (PBS) to remove floating bacteria on the surface of plate. The biofilm above the steel plate was observed by SEM (Fig. 1).

Surgical procedure. Before surgery, the NZW rabbits were fasted for $12 \mathrm{~h}$. They were anaesthetised using an intramuscular injection of $3 \%$ pentobarbital sodium solution $(1 \mathrm{ml} / \mathrm{kg})$ in combination with Xylazine Hydrochloride Injection $(0.1 \mathrm{ml} / \mathrm{kg})$.

The surgery was performed under strict aseptic conditions. The surgical procedure for implant fixation was done using a direct lateral approach to the middle femur as described previously. After skin disinfection using povidone-iodine (anerdian), a 3 4 cm long incision was made at the lateral aspect of the middle right femur. Blunt separation was done along the muscle gap to completely expose the femoral shaft. The pre-bent plate (with or without biofilm, depending upon the animal group) was rinsed with PBS 3 times and placed in front of the femoral shaft and fixed with four screws. Then a fracture was created with a $1 \mathrm{~mm}$ diameter wire saw at the area between the second and third screws. The wound was rinsed with saline, closed in layers. Postoperative monitoring extended until the animals could stand on their own feet and eat and drink as well.

$X$-ray and micro-CT. X-rays of the femur were taken in all animals at day $0,7,14$ and 21 post-surgery, to confirm the osteotomy and the inflammatory changes of bone. After the animals were sacrificed and the plates were removed on day 21 postoperatively, Micro-CT scan was performed on the femur in order to observe and compare the periosteal reaction, callus formation, osteolytic destruction and absorption between the two groups.

Scanning electron micrography (SEM). After the animals were sacrificed on day 21, all the plates were removed and washed 3 times with PBS. Six plates in each group (group 1: 6 plates, group 2: 6 plates) were fixed with $2.5 \%$ glutaraldehyde. After vacuum drying and sputter-coating with gold, the specimens were assessed by a scanning electron microscope operated (HITACHI S-3000N, Japan) at $5 \mathrm{kV}$. Through SEM, we directly compared the biofilm growth on implant surface in the two groups.

Confocal laser scanning microscopy (CLSM). The remaining plates (group 1: 6 plates, group 2: 6 plates) were subjected to bacterial semi-quantitative analysis using LIVE/DEAD ${ }^{\circledR}$ Biofilm Viability Kit (FilmTracer ${ }^{\mathrm{TM}}$, USA) by a confocal laser scanning microscopy (Fluoview FV10i, Olympus). Ultrasonication was utilized to release the bacteria in biofilms on the surface of the plate (11). Directly after retrieval, plates were placed in a $15 \mathrm{ml}$ centrifuge tube containing $5 \mathrm{ml}$ PBS. All the tubes were vortexed for 30 sec using a Vortex-Genie and then subjected to sonication at $40 \mathrm{kHz}$ in a ultrasound bath for $5 \mathrm{~min}$, followed by additional vortexing for $30 \mathrm{sec}$. Ultrasonication at this frequency and duration has previously been demonstrated not to affect the viability of Staphylococcus aureus (12). The solution was centrifuged at $3,000 \mathrm{rpm}$ for $5 \mathrm{~min}$ and the supernatant discarded. Then $200 \mu \mathrm{l}$ of staining solution was added into the solution. The sample was incubated for 20-30 min at room temperature, protected from light. After centrifugation by addition of $5 \mathrm{ml}$ of PBS again, the supernatant was discarded; the solution was subsequently mixed with $5 \mathrm{ml}$ PBS and $300 \mu \mathrm{l}$ of the solution was observed in a confocal dish with CLSM finally. The images were processed by software (ImageJ) and transformed into optical density or area for data analysis.

Histology. After Micro-CT, the femurs were cut according to the length of the plate and decalcified with EDMA before being dehydrated with increasing concentrations of ethanol and embedded in paraffin. Transverse slices $(4 \mu \mathrm{m})$ were made through the screw holes in the bone and stained with Hematoxylin \& Eosin (HE). The sections were examined for inflammatory cells, necrotic tissue, and bone destruction. Specific parameters were assessed on the basis of a modified Smeltzer's grading scale (10). Each parameter was scored on a five-point scale (0-4), with 4 representing the most severe evidence. All individual sections were scored by an independent, outside, blinded observer.

Statistical analysis. Histology scores obtained for each individual parameter were averaged to obtain a composite score for each rabbit. SPSS 20 (IBM, USA) was used for the statistical analyses. The data were checked for homogeneity of variance using levene's test. Differences between groups were determined by independent-sample t test (Student's t test) for parametric two-tailed significance. The significance level was determined at $\mathrm{P}<0.05$. Graphical representation of the data was performed in GraphPad Prism 6 (GraphPad, USA). 


\section{Results}

Clinical assessment. All animals were sacrificed at day 21 (Fig. 2). All animals in groups 2 showed signs of soft tissue and bone infection with significant swelling, pus formation and local tissue destruction (Fig. 2E). Three different points of the pus were taken and painted on the agar plates. After cultured at $37^{\circ} \mathrm{C}$ for $18 \mathrm{~h}$, the plates on which colonies appeared were sent to a clinical laboratory at Nanfang Hospital. Its automatic bacterial identification instrument showed Staphylococcus aureus. Clear instability of the plates and screws, a large amount of reactive callus formation and no clinical fracture healing at the former fracture site were observed in the 10 animals with biofilm infected. In group 1 without biofilm all the animals showed stable plate fixation with a moderate amount of healing callus.

$X$-ray. The X-ray pictures showed stable internal fixation without loosening on days 0 and 7, basically the same. The X-ray results showed the rabbits without biofilm (group 1) had no significant infection within 21 days, but only mild periosteal reaction with no obvious infection at the third week (Fig. 3). Biofilm-infected rabbits (group 2) showed only mild periosteal reaction on day 14 (Fig. 3E) which developed into diffuse osteomyelitis in a week's time. On day 21, significant osteolysis appeared around the implant in the rabbits of group 2 with severe periosteal reaction away from the fracture (Fig. 3F).

Micro-CT. In both infection and non-infection groups, significant bone callus formation was observed (Fig. 4). In contrast to the control group where the callus gathered around the fracture, the callus in the infected group was far away from the fracture site [Fig. 4(a, b)]. This is because the severity of inflammation varies from the proximal to the distal part of a fracture. Studies have shown that inflammation at the distal part of the fracture may be milder than that at the proximal part (13), leading to more obvious callus growth at the distal part. As we all know, the effect of inflammation on the bone-formation is bi-directional. Mild inflammatory response may stimulate osteogenesis while severe inflammatory reaction inhibits or even destroys osteogenesis (14). The cross-sectional images showed although the two groups had callus formation outside the cortex, destruction of the cortex dissolved occurred dramatically in the infection group [Fig. 4(c, f)].

Scanning electron micrography. Bacterial biofilm formed on the steel plate after $48 \mathrm{~h}$ culture in vitro; colonies of coccoid bacteria were embedded in or under an extracellular matrix which was considered as a bacterial biofilm (Fig. 1). The same results were found in the biofilm-infected group, but the area of biofilm was significantly increased. However, all the other plates in the group without bacterial contamination were free of biofilm formation.

Confocal laser scanning microscopy. LIVE/DEAD ${ }^{\circledR}$ Biofilm Viability Kit (FilmTracer ${ }^{\mathrm{TM}}$, USA) utilizes mixtures of the $\mathrm{SYTO}^{\circledR} 9$ green fluorescent nucleic acid stain and the red-fluorescent nucleic acid stain, propidium iodide. It allows researchers to distinguish live and dead bacteria quickly, without waiting for growth plate results. Because of the opaque steel plate, only the edge of the screw hole could be observed to

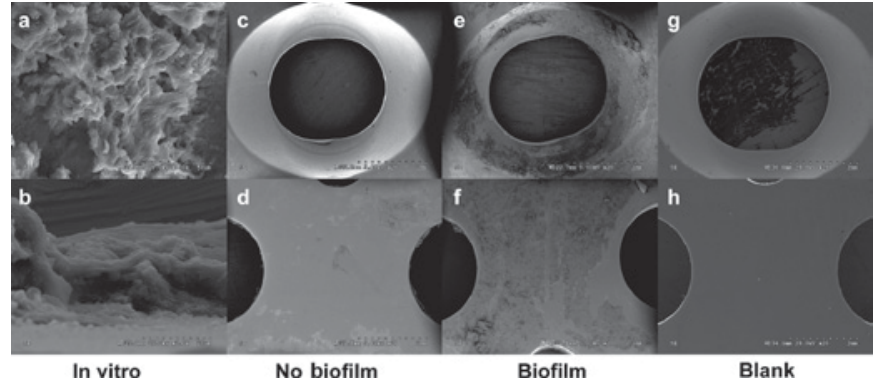

Figure 1. SEM images of a bacterial biofilm growing on a steel plate. Bacterial biofilm formed on the steel plate after $48 \mathrm{~h}$ culture in vitro, with planktonic bacteria adhering to the extracellular matrix of biofilm (A, B). Surface conditions of the steel plate were shown after 3 weeks in vivo $(\mathrm{C}-\mathrm{F})$. Compared with the control group $(\mathrm{C}, \mathrm{D})$, abundant biofilm structure appeared on the surface in the biofilm group (E, F). (Magnifications: a: 1000x, b: 2000x, c-f: 20x). Blank plates $(\mathrm{G}, \mathrm{H})$.

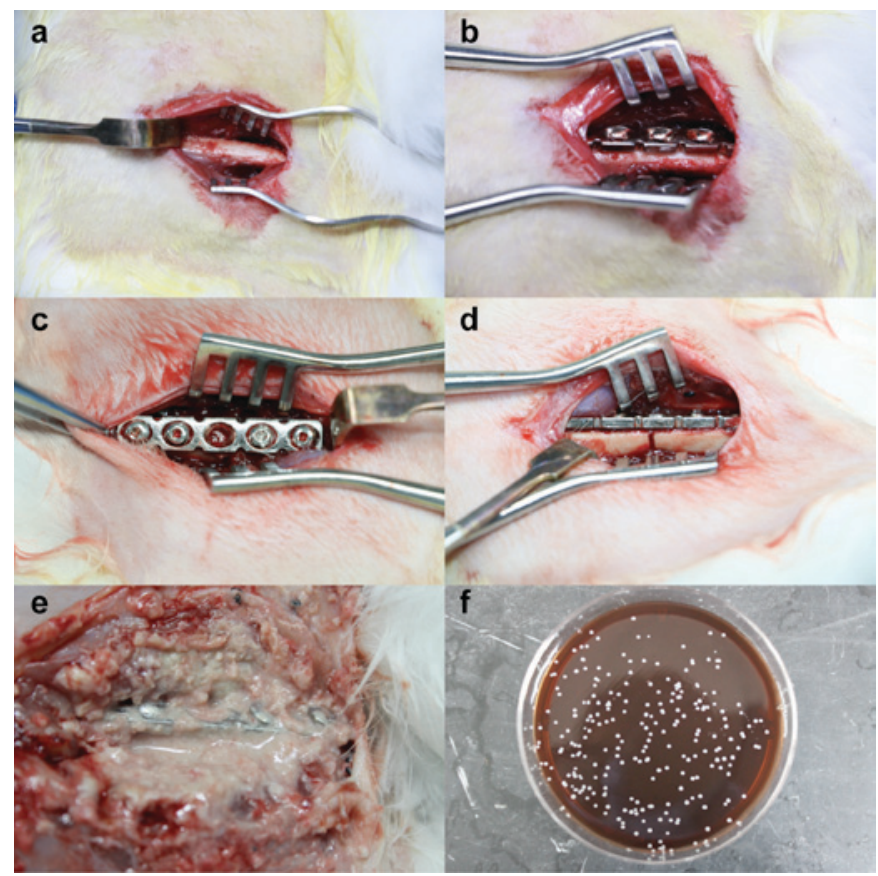

Figure 2. Photographs of the animal surgical procedure (A-D). The right femur was exposed (B) and the pre-bent plate was fixed in front of the femur (C), before a fracture was created (black arrow) (D). The plate in biofilm group was found soaked in the pus at 21 days (E), and $S$. aureus could be cultured from the pus $(\mathrm{F})$.

find that live bacteria became green in fluorescence and dead red (Fig. 5). Thus, we separated the bacterial biofilm from the plate by ultrasonication to count the area of the bacteria biofilm (Fig. 5D). There were evident statistical differences regarding the biofilm area between the two groups $(\mathrm{P}<0.001)$ (Fig. 6).

Histology. Sections stained with H\&E showed an observable difference between Group 1 and Group 2 rabbits. Cortical bone growth and inflammatory response were not decisive indicators of infection as the results suggested that they could be caused by surgical trauma and infection; however, there was a notable difference in the degree of response between the two groups in bone morphology. Those that had infection showed a large amount of inflammatory cell infiltration with 


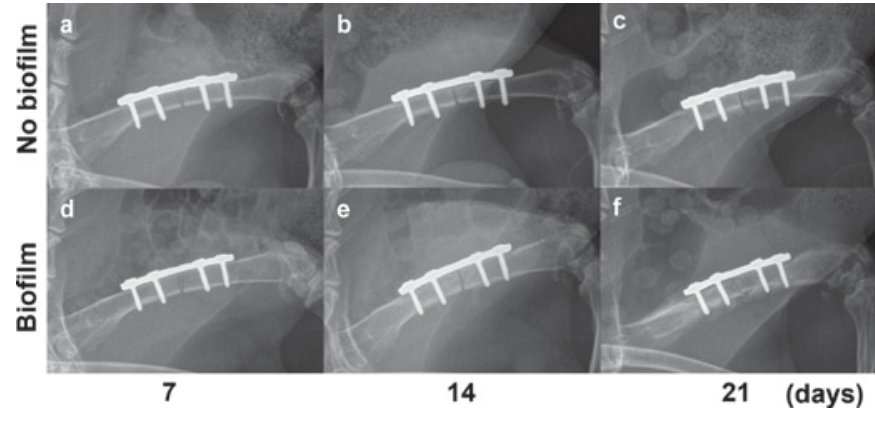

Figure 3. (A-D) Radiological changes in the right femur fixed with a steel plate. On days 7 and 14, the two groups did not show significant differences in the imaging (A, B and D); only mild periosteal reaction was found in $(\mathrm{C})$ the control group on day 21 and (E) the biofilm group on day 14. (F) By contrast, obvious osteomyelitis emerged on day 21 .

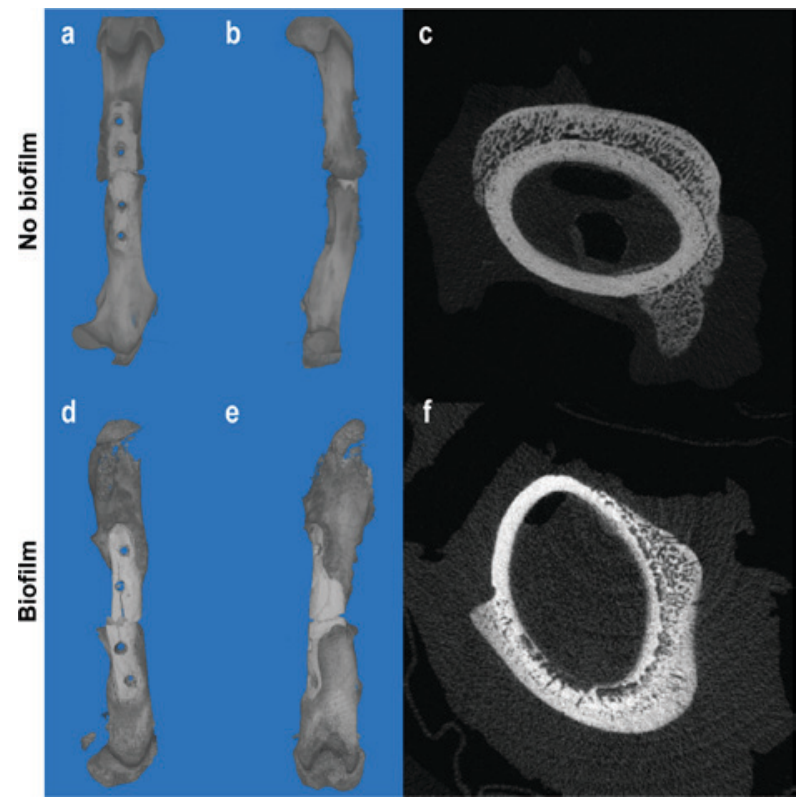

Figure 4. (A-F) Tomography and three-dimensional reconstruction images of the femur in two groups on day $21.3 \mathrm{D}$ reconstruction images revealed significant bone callus formation around the fracture site in the control group (A and B), while a large amount of callus formed away from the fracture site in the biofilm-infected group (D and E). The cortical bone in the infected group showed obvious corrosion and absorption (F), while in the control group it remained intact (C).

signs of 'moth eaten' bone that had jagged edges due to resorption/bacterial presence, whereas those with a small amount of inflammatory cells due to surgical trauma had little indication that resorption was occurring (Fig. 7). More specifically, scattered cell-free sequestrum was found in sections in the infected group (Fig. 7D). The histological scores of the two groups were significantly different $(\mathrm{P}<0.001)$ (Fig. 8).

\section{Discussion}

The above experimental results demonstrate that all the animals in the infection group presented with typical signs of osteomyelitis and the difference between the experimental and control groups was obvious. This animal model is stable, reproducible and suitable for the study on treatment strategies for osteomyelitis and antibacterial capacity of different

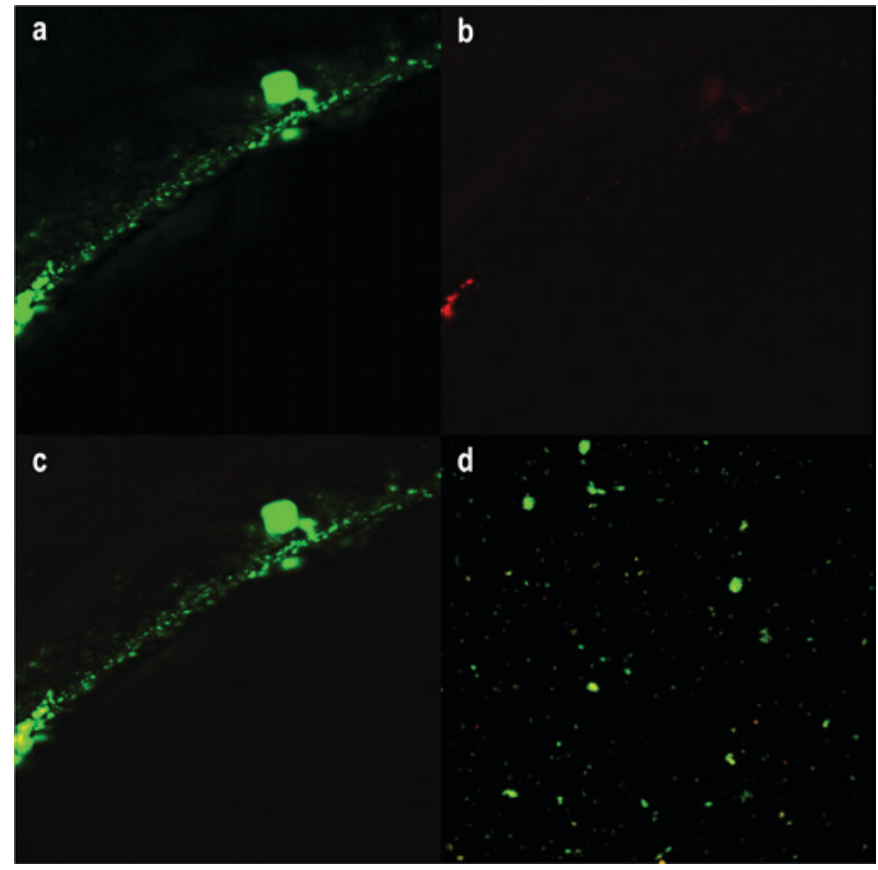

Figure 5. The CSLM images of bacterial biofilm on the edge of steel plate $(\mathrm{A}-\mathrm{C})$. Pictures a and $\mathrm{b}$ were in different excitation light, and $\mathrm{c}$ was the combination of the two. Live bacteria in the excitation light emitted green fluorescence while dead bacteria showed red. By ultrasonication, the biofilm could be removed from implants (D).

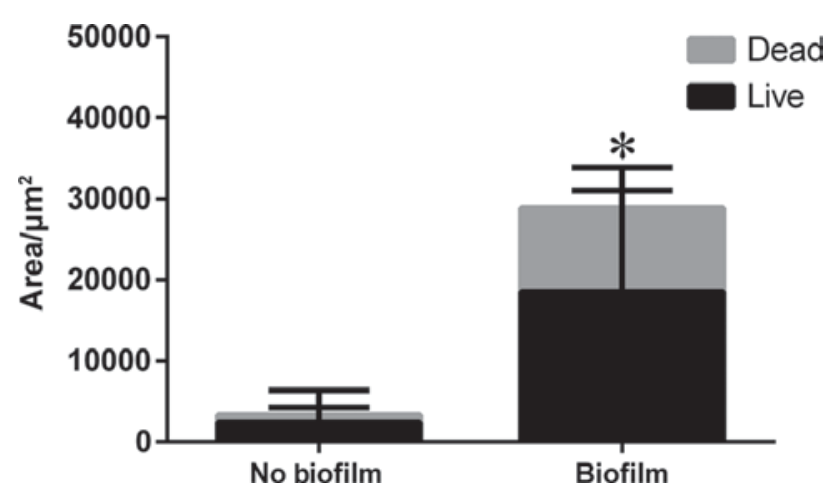

Figure 6. Area of the biofilm included live and dead bacteria in the two groups. ${ }^{*} \mathrm{P}<0.001$.

materials, and even suitable for observation of fracture healing. In addition to improved repeatability and stability, our model also has an advantage of reduced modeling time because it takes less time for bacteria to proliferate and adhere to the surface of the plate and form a mature biofilm in the presence of human body immune reaction. In the present study, implant-related osteomyelitis developed within 21 days in all the surviving rabbits in group 2 contaminated with biofilm bacterial inoculum, much sooner than 4-6 weeks reported by other relevant studies $(3,15,16)$.

Since stability of internal fixation plays an important role in reduction and fracture healing, the absence of stability will have a negative effect on the outcomes of the experiments using these animal models. Choice of steel plate fixation may be a desirable solution to the absence of stability, but most of the current animal models do not use internal fixation with steel plate because osteotomy is not performed due 


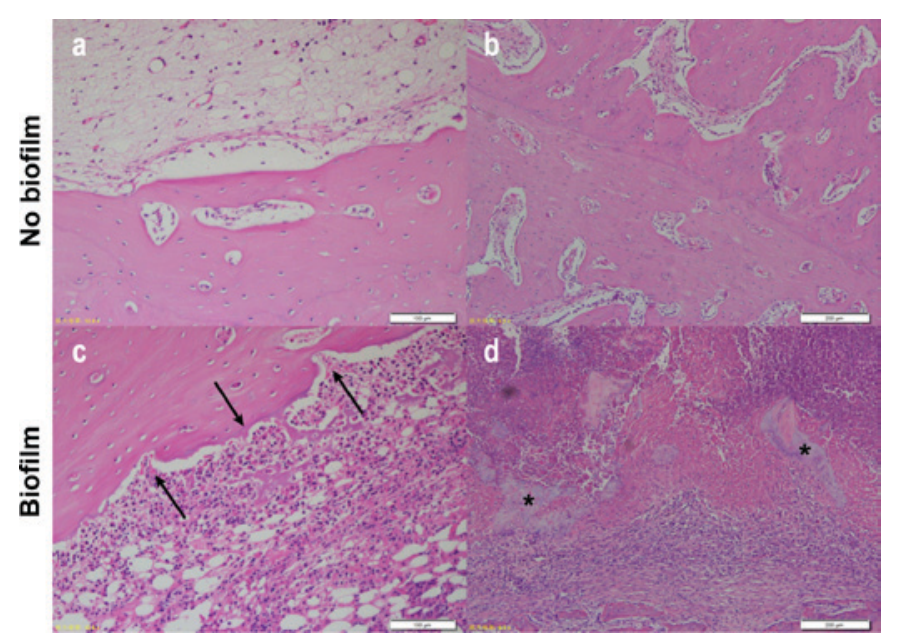

Figure 7. Images of H\&E-stained bone sections. In contrast to group 1 (A and B), group 2 had more severe infiltration of inflammatory cells and destruction of cortical bone (arrows) (C). Scattered sequestrum was found in the group contaminated with biofilm (asterisks) (D).

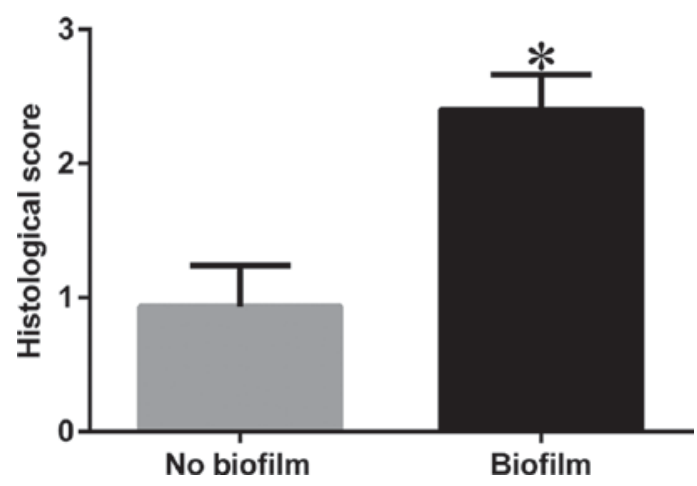

Figure 8. Histologic scores. $\mathrm{P}<0.001$.

to insufficient rotational stability (17-20). Currently, intramedullary fixation with a K-wire is widely used in animal models of implant-related osteomyelitis (16). However, due to limitations in animal size and equipment, such a fixation method apparently fails to provide the stability required for fracture healing, and must have an uncertain impact on the occurrence and development of infection. This may lead to unsatisfactory experimental results, no matter osteotomy is performed or not. Moreover, in clinic, surgeons usually prefer plate fixation to intramedullary fixation for patients with open fracture who still have a high possibility of infection despite thorough debridement. Taking the above aspects into account, the present study chose plate fixation to provide stability of fracture, instead of intramedullary fixation. We didn't create a fracture before pre-bent plate was fixed, due to the difficulty of anatomical reduction, higher surgical failure rate, as well as higher mortality of animals. Besides, although the tibia is mainly utilized in the open fracture models $(3,16,21)$, the femoral shaft was selected as the location for plate fixation in the present study because in anatomical morphology the rabbit femoral shaft is flat with little pre-arch, unlike the triangular prism of the tibia.

Most animal models of osteomyelitis primarily use an initial inoculum of planktonic bacterial cells $(15,19,22,23)$, with an expectation that these planktonic cells will attach to the surface of medical devices or to the surrounding tissue and subsequently form a biofilm. However, according to Williams et al (9), there may be three major limitations which may accompany the use of bacteria in animal models of osteomyelitis. (1) Planktonic bacterial cells are more likely to be cleared by the immune system than biofilm bacteria, and immune systems of animals are innately more powerful than those of humans. (2) Planktonic bacteria are more susceptible to antibiotics than cells residing in biofilm. $>1,000$ times of the minimum inhibitory concentration are required to treat biofilm infections (24). (3) The possibility exists for planktonic bacteria to be diluted by animal body fluids, which may result in insufficient concentration to attach to tissue or medical devices. When we model osteomyelitis and perform early intervention with antibiotics, the above limitations may lead to inconsistent results, affecting the repeatability of infection development as well as the effect of antibiotic treatment.

Since animal models with good reproducibility and stability are designed to solve clinical problems, they should simulate clinical settings as closely as possible. The osteomyelitis animal model is an artificial confrontation between the host immune system and bacterial virulence. The pure cultures that have proven so valuable in the laboratory are virtually absent in nature: $>99.9 \%$ of bacteria exist in heterogeneous communities called biofilms (25). As they can form on nearly every surface, the bacteria on them, rather than floating bacteria, are in action when contamination occurs. Animal models inoculated with floating bacteria still need a process of biofilm formation, but host immunity will have a strong resistance to this process. This is the reasons why this modeling method is unstable. Low concentrations of bacterial suspensions are not sufficient to overcome host immunity, but high concentrations are likely to overwhelm the host immunity completely, leading to high mortality. In order to overcome these defects, we avoided destructing host immunity by inoculating a biofilm on the surface of an in vitro steel plate. We believe that the biofilm bacteria which are in a relatively dormant state will not cause a violent confrontation with host immunity so that the host has enough time to get adapted to the gradual escalation of bacterial virulence.

It has to be noticed that our method for quantifying bacteria is not by conventional bacterial culture but by combining CLSM with ImageJ software. This was because many studies confirmed unsatisfactory effect of bacterial culture on the biofilm (1). The positive rate of traditional culture is low and bacteria wrapped in the biofilm cannot be cultured. The first method of semi-quantitative determination of biofilm was developed by Christensen et al, measuring the absorbance of biofilm stained with crystal violet (26). After continuous improvement, spectrophotometry has become a widespread method for biofilm evaluation, but the scarce specificity still limits its application (27).

Despite the improvements, we also have shortcomings in our study. As a result of the absence of comparison with the inoculation of floating bacteria, we can not get a definite result about any difference with this model between planktonic vs. biofilm inocula, which is a serious deficiency in our study. We will continue to explore their differences in our further study. Although SEM is one of the most important 
tools to observe the structure of bacterial biofilm, it can be used to observe only the surface but not the internal composition and structure. Currently, no objective measurements are available to distinguish the stages of biofilm formation. This is indeed a limitation that is worth exploring. Identifying early and mature biofilms and comparing them with floating bacteria will be a chief concern of our future study. Since the concern of this present study was to establish a novel animal model with biofilms as initial inoculation to explore the feasibility to improve the stability and repeatability of a currently conventional animal model of osteomyelitis, but not to compare the intervention factors for fracture fixation, we did not establish an intramedullary fixation as another control group. Moreover, Long-axial Multiplanar Reconstruction (MPR) images are better and more realistic to show the callus and osteomyelitis than three-dimensional reconstruction. Besides, our model only involved biofilm as initial inoculum of S. aureus after plate fixation. In fact, the occurrence and development of osteomyelitis is related to a number of factors, such as material of the plate, species of the bacterium, virulence of the bacterium and immune status of the host. Although it appears that S. aureus will still be the primary pathogen for osteomyelitis for a long time, the effect of other pathogens should not be ignored, such as S. epidermidis, P. aeruginosa and polymicrobial ones. Different bacteria may lead to changes in the pathophysiology, making the experimental results varied.

CLSM is one of the most sensitive and specific methods for analysis of biofilm structures, and for determination of bacterial survival as well. However, limited by the confocal microscopy (Fluoview FV10i, Olympus) we used, most areas of the steel plate could not be observed, except for the edges irradiated by the laser. Therefore, we used ultrasonic method, as previously mentioned, to remove the biofilm from the plates before we performed a semi-quantitative analysis with CLSM. However, our CLSM remains a very worthwhile attempt; some scientists have made it possible to measure biomass volume with CLSM improved by computer technology (28). We hope to make up for this shortcoming in the future experiment.

\section{Conclusion}

The results show that we have successfully established a rabbit model of infection after open fracture fixation. We believe that our model is an improvement of the previous model. The primary benefit of this model lies in its stability and repeatability, and its good simulation of clinical conditions as well. It can be used to replace traditional models, but does not seem to have a very direct impact on how to improve clinical care and patient outcomes. However, when the stability and repeatability is improved, our new model helps to produce more accurate experimental results, though the effects of our improved model are not obvious at present. We hope that this animal model could be verified and further improved by more studies.

\section{Acknowledgements}

The authors thank Professor Liang and all the staff in the Key Laboratory of Bone and Cartilage Regenerative Medicine,
Nanfang Hospital of Southern Medical University. This work was supported by the National Natural Science Foundation of China [grant nos. 2016B090913004/201508020035].

\section{References}

1. Arciola CR, Campoccia D, Ehrlich GD and Montanaro L: Biofilm-based implant infections in orthopaedics. Adv Exp Med Biol 830: 29-46, 2015

2. Metsemakers WJ, Kuehl R, Moriarty TF, Richards RG, Verhofstad MH, Borens O, Kates S and Morgenstern M: Infection after fracture fixation: Current surgical and microbiological concepts. Injury, Sep 11, 2016 (Epub ahead of print).

3. Worlock P, Slack R, Harvey L and Mawhinney R: An experimental model of post-traumatic osteomyelitis in rabbits. Br J Exp Pathol 69: 235-244, 1988

4. Reizner W, Hunter JG, O'Malley NT, Southgate RD, Schwarz EM and Kates SL: A systematic review of animal models for Staphylococcus aureus osteomyelitis. Eur Cell Mater 27: 196-212, 2014

5. Donné J and Dewilde S: The challenging world of biofilm physiology. Adv Microb Physiol 67: 235-292, 2015.

6. Costerton JW: Cystic fibrosis pathogenesis and the role of biofilms in persistent infection. Trends Microbiol 9: 50-52, 2001.

7. Montanaro L, Speziale P, Campoccia D, Ravaioli S, Cangini I, Pietrocola G, Giannini S and Arciola CR: Scenery of Staphylococcus implant infections in orthopedics. Future Microbiol 6: 1329-1349, 2011.

8. Trampuz A and Zimmerli W: Diagnosis and treatment of infections associated with fracture-fixation devices. Injury 37 (Suppl 2): S59-S66, 2006.

9. Williams DL and Costerton JW: Using biofilms as initial inocula in animal models of biofilm-related infections. J Biomed Mater Res B Appl Biomater 100: 1163-1169, 2012.

10. Williams DL, Haymond BS, Woodbury KL, Beck JP, Moore DE, Epperson RT and Bloebaum RD: Experimental model of biofilm implant-related osteomyelitis to test combination biomaterials using biofilms as initial inocula. J Biomed Mater Res A 100: 1888-1900, 2012

11. Trampuz A, Piper KE, Jacobson MJ, Hanssen AD, Unni KK, Osmon DR, Mandrekar JN, Cockerill FR, Steckelberg JM, Greenleaf JF and Patel R: Sonication of removed hip and knee prostheses for diagnosis of infection. N Engl J Med 357: 654-663, 2007.

12. Monsen T, Lövgren E, Widerström M and Wallinder L: In vitro effect of ultrasound on bacteria and suggested protocol for sonication and diagnosis of prosthetic infections. J Clin Microbiol 47: 2496-2501, 2009.

13. Rochford ET, Sabaté Brescó M, Zeiter S, Kluge K, Poulsson A, Ziegler M, Richards RG, O'Mahony L and Moriarty TF: Monitoring immune responses in a mouse model of fracture fixation with and without Staphylococcus aureus osteomyelitis. Bone 83: 82-92, 2016.

14. Glass GE, Chan JK, Freidin A, Feldmann M, Horwood NJ and Nanchahal J: TNF-alpha promotes fracture repair by augmenting the recruitment and differentiation of muscle-derived stromal cells. Proc Natl Acad Sci USA 108: 1585-1590, 2011.

15. Kishor C, Mishra RR, Saraf SK, Kumar M, Srivastav AK and Nath G: Phage therapy of staphylococcal chronic osteomyelitis in experimental animal model. Indian J Med Res 143: 87-94, 2016.

16. Odekerken JC, Arts JJ, Surtel DA, Walenkamp GH and Welting TJ: A rabbit osteomyelitis model for the longitudinal assessment of early post-operative implant infections. J Orthop Surg Res 8: 38, 2013.

17. Moriarty TF, Campoccia D, Nees SK, Boure LP and Richards RG: In vivo evaluation of the effect of intramedullary nail microtopography on the development of local infection in rabbits. Int $\mathbf{J}$ Artif Organs 33: 667-675, 2010.

18. Norden CW: Experimental osteomyelitis. I. A description of the model. J Infect Dis 122: 410-418, 1970.

19. Shiels SM, Bedigrew KM and Wenke JC: Development of a hematogenous implant-related infection in a rat model. BMC Musculoskelet Disord 16: 255, 2015.

20. Arens S, Kraft C, Schlegel U, Printzen G, Perren SM and Hansis M: Susceptibility to local infection in biological internal fixation. Experimental study of open vs minimally invasive plate osteosynthesis in rabbits. Arch Orthop Trauma Surg 119: 82-85, 1999. 
21. Alt V, Lips KS, Henkenbehrens C, Muhrer D, Oliveira Cavalcanti MC, Sommer U, Thormann U, Szalay G, Heiss C, et al: A new animal model for implant-related infected non-unions after intramedullary fixation of the tibia in rats with fluorescent in situ hybridization of bacteria in bone infection. Bone 48: 1146-1153, 2011.

22. Svensson S, Trobos M, Hoffman M, Norlindh B, Petronis S, Lausmaa J, Suska F and Thomsen P: A novel soft tissue model for biomaterial-associated infection and inflammation-Bacteriological, morphological and molecular observations. Biomaterials 41: 106-121, 2015.

23. dos Reis JA Jr, de Carvalho FB, Trindade RF, de Assis PN, de Almeida PF and Pinheiro AL: A new preclinical approach for treating chronic osteomyelitis induced by Staphylococcus aureus: In vitro and in vivo study on photodynamic antimicrobial therapy (PAmT). Laser Med Sci 29: 789-795, 2014.

24. Gnanadhas DP, Elango M, Janardhanraj S, Srinandan CS, Datey A, Strugnell RA, Gopalan J and Chakravortty D: Successful treatment of biofilm infections using shock waves combined with antibiotic therapy. Sci Rep 5: 17440, 2015.
25. Wimpenny J, Manz W and Szewzyk U: Heterogeneity in biofilms. FEMS Microbiol Rev 24: 661-671, 2000.

26. Christensen GD, Simpson WA, Younger JJ, Baddour LM, Barrett FF, Melton DM and Beachey EH: Adherence of coagulase-negative staphylococci to plastic tissue culture plates: A quantitative model for the adherence of staphylococci to medical devices. J Clin Microbiol 22: 996-1006, 1985.

27. Vassena C,Fenu S, GiulianiF, Fantetti L, Roncucci G,Simonutti G, Romanò CL, De Francesco R and Drago L: Photodynamic antibacterial and antibiofilm activity of RLP068/Cl against Staphylococcus aureus and Pseudomonas aeruginosa forming biofilms on prosthetic material. Int J Antimicrob Agents 44: 47-55, 2014

28. Drago L, Agrappi S, Bortolin M, Toscano M, Romanò C and De Vecchi E: How to study biofilms after microbial colonization of materials used in orthopaedic implants. Int J Mol Sci 17: 293, 2016. 